\title{
ENLARGING A FREE SUBGROUP OF A SYMMETRIC GROUP FREELY
}

\author{
FRANKLIN HAIMO AND MARVIN TRETKOFF
}

\begin{abstract}
Free subgroups, the elements of which have ample cycles in their cycle decompositions, are enlarged to free subgroups of an infinite symmetric group by the specific construction of one more free generator.
\end{abstract}

1. Introduction. Investigations of some open Riemann surfaces lead to the question of whether a free subgroup on a free basis in an infinite symmetric group can be extended to a free subgroup by augmenting that free basis by one more generator ([2], [5]). Suppose that the free rank is infinite but of smaller cardinality than that of the permuted set on which the symmetric group acts. Consider any nontrivial element of the free group as a permutation, and suppose that our embedding is such that the cardinal of the set of nontrivial cycles in the disjointcycle decomposition (d.c.d.) of that element always exceeds the free-rank cardinal. Then we shall show that the desired augmentation is always possible.

We well order the set of nontrivial elements of the free group and, by transfinite induction, construct an indexed set of moved points. After suitably reindexing that set, we use its members as "anchor points" for a disjoint collection of finite cycles. From this disjoint collection our new, free generator emerges.

A subset $L$ of a group $G$ generates a subgroup denoted by $\langle L\rangle$. The cardinal of a set $M$ is written as $|M|$. If $M$ is any nonvoid set, and if Sym $M$ is the symmetric group on $M$, then each nontrivial member $x$ of Sym $M$ has disjoint-cycle decomposition, d.c.d.(x), a set of disjoint, nontrivial cycles of members of $M$, where the nontrivial transitivity classes of $\langle x\rangle$, the subgroup of Sym $M$ generated by $x$, are precisely the sets of moved points of these various cycles [6]. Recall that if $M$ is infinite, an element of Sym $M$ need not, contrary to the situation in the finite case, be the product of the cycles in its d.c.d. ([4], [7]). Symbols like $\mathbf{Z}_{>n}$ and $\mathbf{Z}_{>n}$ denote the sets of integers respectively $\geqslant n$ and $>n$.

Much has been done (e.g., [1]) in showing that free groups of quite high infinite rank can be injected into symmetric groups that permute sets of only moderately large cardinality. But our task is almost quite the opposite: we start with moderately large free groups embedded into very large symmetric groups and try for only very modest free enlargements.

The authors express their gratitude to the referee for most helpful suggestions, to Michael F. Singer for perceptive comments, and to their various host institutions,

Received by the editors June 11, 1979 and, in revised form, August 21, 1980.

1980 Mathematics Subject Classification. Primary 20E05, 20B35. 
The Institute for Advanced Study and Princeton University, for generous hospitality.

2. The existence of embeddings. Let $\mathfrak{b}$ and $\mathfrak{h}$ be infinite cardinals where $\mathfrak{h}>\mathfrak{b}$. In a symmetric group Sym $H$ of degree $\mathfrak{h}$ is it possible to find at least one subgroup that is a free group of free rank $\mathfrak{b}$ ? And, can the embedding be done in such a way that, for each nontrivial member of the free subgroup, the d.c.d. in Sym $H$ will have more than $\mathfrak{b}$ cycles? Should such an embedding exist, it will be called an $(\mathfrak{h}, \mathfrak{b})$-ample embedding.

LEMMA. Let $S$ be a symmetric group of degree $\mathfrak{h}$, a noncountable cardinal. For each infinite cardinal $\mathfrak{b}<\mathfrak{h}$ there exists at least one free subgroup $A$ of $S$ of free rank $\mathfrak{b}$ such that the embedding of $A$ in $S$ is $(\mathfrak{h}, \mathfrak{b})$-ample.

Proof. Let $H$ be an index set of cardinal h. Construct a family $\left\{M_{h}\right\}$ of mutually disjoint sets $M_{h}$, one for each $h \in H$, where each $\left|M_{h}\right|=\mathfrak{b}$. Let $S_{h}=\operatorname{Sym} M_{h}$, let $F$ be a free group of free rank $\mathfrak{b}$, so that $|F|=\mathfrak{b}$, and let $\psi_{h}$ be the Cayley right representation of $F$ in $S_{h}$. If $M=\cup_{h \in H} M_{h}$, each $S_{h}$ is embedded in an obvious way in $\operatorname{Sym} M$, and $|M|=\mathfrak{h}$. If $b \in F \backslash\left\{1_{F}\right\}, \psi_{h}(b)$ will have $\mathfrak{b}$ infinite cycles in its d.c.d. in $S_{h}$. Let $\psi_{h}^{\prime}$ be $\psi_{h}$ with its codomain extended from $S_{h}$ to Sym $M$, and let $Q_{b}=\cup_{h}$ d.c.d. $\left(\psi_{h}^{\prime}(b)\right)$. Then the set $Q_{b}$ consists of $\mathfrak{h}$ disjoint infinite cycles of members of $M$. There exists a unique $b^{\prime} \in \operatorname{Sym} M$ such that d.c.d. $\left(b^{\prime}\right)=Q_{b}$. Let $\psi$ be the map from $F$ to $\operatorname{Sym} M$ that carries each $b \in F \backslash\left\{1_{F}\right\}$ to $b^{\prime} \in \operatorname{Sym} M$ and carries $1_{F}$ to the unity of Sym $M$. Then $\psi$ embeds $F$ monomorphically into Sym $M$, and this embedding is $(\mathfrak{h}, \mathfrak{b})$-ample.

Since the symmetric group $S$ of the hypothesis is of degree $\mathfrak{h}$, it is permutationgroup isomorphic to $\operatorname{Sym} M$. Thus, there must exist a free subgroup $A$ of $S$ of free rank $\mathfrak{b}$ such that the embedding is $(\mathfrak{h}, \mathfrak{b})$-ample.

Observe that the embeddings used in the proof above are "superample" in that in each pertinent d.c.d. we get $\mathfrak{h}$ infinite cycles (and no finite cycles), not just more than $\mathfrak{b}$ cycles.

3. The principal result. If $\zeta \in \operatorname{Sym} H, s(\zeta)$, the support of $\zeta$, is the set of all points in $H$ that are not fixed by $\zeta$.

THEOREM. Let $A$ be a free group on a free basis $B$ of infinite cardinality. Also, let $A$ be a subgroup of a symmetric group $\operatorname{Sym} H$, where $|H|>|B|$, in such a way that the embedding is $(|H|,|B|)$-ample. Then there exists at least one $\beta \in(\operatorname{Sym} H) \backslash A$ with the following properties:

(1) d.c.d. $(\beta)$ has only finite cycles, and $\beta$ is fixed-point free;

(2) $\langle B \cup\{\beta\}\rangle$ is a free subgroup of Sym $H$ on the set $B \cup\{\beta\}$ of free generators and properly includes $A$.

Proof. Let $\mathfrak{b}=|B|(=|\langle B\rangle|=|A|)$, and let $\mathfrak{h}=|H|$. Well order $A \backslash\left\{1_{A}\right\}$, calling its members $w_{0}, w_{1}, \ldots, w_{\mu}, \ldots$, indexed by ordinals $\mu$ in some initial segment $M$ of ordinals. Let $D_{\mu}=$ d.c.d. $\left(w_{\mu}\right)$, so that, by hypothesis, $\mathfrak{h}>\left|D_{\mu}\right|>\mathfrak{b}$. 
Let $T_{0}$ be an index set of cardinality $\mathfrak{b}$, and let $\rho_{0}$ be a monic function from $T_{0}$ into $D_{0}$. For each $\tau_{0} \in T_{0}$ let $\rho_{0}\left(\tau_{0}\right)=C_{\tau_{0}}$, a cycle in $D_{0}$. Let $C_{0}=\left\{C_{\tau_{0}} \mid \tau_{0} \in T_{0}\right\}$, the range of $\rho_{0}$, so that $\left|C_{0}\right|=\mathfrak{b}$.

Suppose that $\nu \in M$ and that, for each $\mu \in M, \mu<\nu$, sets $T_{\mu}$ have been constructed, as well as monic maps $\rho_{\mu}$ from $T_{\mu}$ into $D_{\mu}$, subject to the following:

(a) each $\left|T_{\mu}\right|=\mathfrak{b}$, and the $T_{\mu}$ are mutually disjoint;

(b) the supports $s\left(\rho_{\mu}\left(\tau_{\mu}\right)\right)$ constitute a family of mutually disjoint sets for all $\mu<\nu$ and all $\tau_{\mu} \in T_{\mu}$.

It is convenient to write $C_{\tau_{\mu}}=\rho_{\mu}\left(\tau_{\mu}\right)$ and $C_{\mu}=\left\{C_{\tau_{\mu}} \mid \tau_{\mu} \in T_{\mu}\right\}$. Observe that each $\left|C_{\mu}\right|=\mathfrak{b}$, so that the disjoint union $\cup_{\tau_{\mu}} s\left(C_{\tau_{\mu}}\right)$ has cardinality $\mathfrak{b}$ for fixed $\mu<\nu$. Let $W_{\nu}=\cup_{\mu<\nu} \cup_{\tau_{\mu}} s\left(C_{\tau_{\mu}}\right)$. Now $|M|=\left|A_{\mu} \backslash\left\{1_{A}\right\}\right|=|A|=|B|=\mathfrak{b}$, so that $\left|W_{\nu}\right|<\mathfrak{b}^{2}$ $=\mathfrak{b}$. But $\left|D_{\nu}\right|>\mathfrak{b}$, and there can be at most $\mathfrak{b}$ cycles in $D_{\nu}$ in which members of $W_{\nu}$ can occur, so that there are more than $\mathfrak{b}$ cycles in $D_{\nu}$ that move no members of $W_{\nu}$. Such cycles will be called $W_{v}$-free.

Let $T_{\nu}$ be a set of cardinality $\mathfrak{b}$, disjoint from the $T_{\mu}$ already specified for the $\mu<\nu$. There exists at least one monic map $\rho_{\nu}$ from $T_{\nu}$ into $D$, such that the range of $\rho_{\nu}$ consists only of $W_{\nu}$-free cycles. As before, $C_{\nu}=\left\{C_{\tau_{\nu}} \mid \tau_{\nu} \in T_{\nu}\right\}$ (where $C_{\tau_{\nu}}=$ $\left.\rho_{\nu}\left(\tau_{\nu}\right)\right)$ has cardinal $\mathfrak{b}$, and the sets $s\left(C_{\tau_{\mu}}\right)$ for all $\mu \leqslant \nu$ and all $\tau_{\mu} \in T_{\mu}$ are mutually disjoint. By transfinite induction we have thus created a set $C$ of disjoint cycles $C_{\tau_{\mu}} \in D_{\mu}$ for all $\mu \in M$ and all $\tau_{\mu} \in T_{\mu}$. We see that $|C|=\mathfrak{b}$.

Choose $u_{\tau_{\mu}}^{\prime} \in s\left(C_{\tau_{\mu}}\right)$, and let $v_{\tau_{\mu}}^{\prime}=u_{\tau_{\mu}}^{\prime} w_{\mu}$. Note that $v_{\tau_{\mu}}^{\prime} \in s\left(C_{\tau_{\mu}}\right)$ and that $u_{\tau_{\mu}}^{\prime} \neq v_{\tau_{\mu}}^{\prime}$. Let $J$ be the set of all these $u_{\tau_{\mu}}^{\prime}$ 's and $v_{\tau_{\mu}}^{\prime}$ 's (all distinct), so that $|\vec{J}|=\mathfrak{b}$.

Let $G$ be a multiplicatively written, infinite cyclic group on the free generator $\gamma$ where $G$ and $A$ as sets have only $1_{G}=1_{A}$ in common. Let $A^{\#}=A * G$, the free product of $A$ and $G$, thus a free group of free rank $\mathfrak{b}$ on the free basis $B \cup\{\gamma\}$. Well order $A^{\#} \backslash\left\{1_{A^{*}}\right\}$, indexing its elements $t_{\alpha}$ by an initial segment $N$ of ordinals $\alpha$. Note that $|N|=\mathfrak{b}$. Each $t_{\alpha}$ has an expansion as a unique product of elements from $A$ and $G$ in that $t_{\alpha}=\omega(\alpha, 1) \gamma^{e(\alpha, 1)} \ldots \omega(\alpha, r(\alpha)) \gamma^{e(\alpha, r(\alpha))}$ where each $\omega(\alpha, j)$ $\in A$ and each $e(\alpha, j)$ is an integer; where $r(\alpha)$ is a positive integer such that if $r(\alpha)>1$ then each $\omega(\alpha, j) \neq 1_{A}$ for $1<j<r(\alpha)$ but where each $e(\alpha, j) \neq 0$ for $1<j<r(\alpha)$; and where $r(\alpha)=1$ implies that $\omega(\alpha, 1) \neq 1_{A}$ or $e(\alpha, 1) \neq 0$. Observe that if $\omega(\alpha, j)=1_{A}$ then $j=1$.

We see that $r$ is a function with domain $N$ and codomain and range $\mathbf{Z}_{>0}$. Let $P$ be that subset of $N \times \mathbf{Z}_{>0}$ which is determined by $r$ upon placing $(\alpha, j) \in P$ if and only if $1<j<r(\alpha)$. Then $e$ is a function with domain $P$ and codomain and range $Z$. Likewise, $\omega$ is a function with domain $P$ and codomain and range $A$. Observe that $|P|=\mathfrak{b}$.

Let $w_{-1}=1_{A}$, and augment $M$ to $M^{\prime}=M \cup\{-1\}$. The function $w$ from $M^{\prime}$ onto $A$ given by $w(\mu)=w_{\mu}$ is a bijection, so that $\Omega=w^{-1} \omega$ (here, functions written to the left of arguments) is a function from $P$ onto $M^{\prime}$ for which $\left|\Omega^{-1}(\mu)\right|=\mathfrak{b}$. The set of all $\Omega^{-1}(\mu)$ 's constitutes a disjoint cover for $P$. Since $\left|\Omega^{-1}(\mu)\right|=\mathfrak{b}=\left|T_{\mu}\right|$ there exists at least one bijection $\phi_{\mu}$ from $\Omega^{-1}(\mu)$ onto $T_{\mu}$. Let $\Phi$ be the bijection from $P \backslash \Omega^{-1}(-1)$ onto the disjoint union $T=\cup_{\mu \in M} T_{\mu}$ given by $\Phi(\alpha, j)=\phi_{\mu}(\alpha, j)$ whenever $(\alpha, j) \in \Omega^{-1}(\mu)$. 
For each $(\alpha, j) \in P \backslash \Omega^{-1}(-1)$ let $u(\alpha, j)=u_{\Phi(\alpha, j)}^{\prime}$, and let $v(\alpha, j)=u(\alpha, j) \omega(\alpha, j)$, which reduces to $v_{\Phi(\alpha, j)}^{\prime}$. Then $u$ is a function from $P \backslash \Omega^{-1}(-1)$ to $H$ with range $U^{\prime}=\left\{u_{\tau_{\mu}}^{\prime} \mid \tau_{\mu} \in T_{\mu}\right.$ and $\left.\mu \in M\right\}$. Since $\Phi$ is a bijection $u$ is monic. Likewise, $v$ is monic, sharing domain $P \backslash \Omega^{-1}(-1)$ and codomain $H$ with $u$ but where $v$ has range $V^{\prime}=\left\{v_{\tau_{\mu}}^{\prime} \mid \tau_{\mu} \in T_{\mu}\right.$ and $\left.\mu \in M\right\}$.

For $\alpha \in N$, let

$$
U^{(\alpha)}= \begin{cases}\{u(\alpha, j) \mid 2<j<r(\alpha)\} & \text { if } \omega(\alpha, 1)=1_{A} \\ \{u(\alpha, j) \mid 1<j<r(\alpha)\} & \text { if } \omega(\alpha, 1) \neq 1_{A}\end{cases}
$$

Similarly, define $V^{(\alpha)}$ by replacing each $u(\alpha, j)$ by $v(\alpha, j)$ in the definition of $U^{(\alpha)}$. Let $U=\cup_{\alpha} U^{(\alpha)}$ and $V=\cup_{\alpha} V^{(\alpha)}$ ( $\alpha$ running over $N$ ), mutually disjoint sets, each a disjoint union of the $U^{(\alpha)}$ 's (respectively, $V^{(\alpha)}$ 's). One readily shows that $U=U^{\prime}$ and $V=V^{\prime}$, so that $U^{\prime} \cup V^{\prime}=J=U \cup V$. We have effectively relabeled the elements of $J$ in a way that will be useful in the construction to come of a free subgroup of Sym $H$ properly including $A$, while the original labeling is convenient in the foregoing, transfinite construction of $J$.

Let $e_{0}$ be the function from $P$ to $\mathrm{Z}$ given by $e_{0}(\alpha, j)=-1$ if $r(\alpha)>j=1$ and $\omega(\alpha, 1) \neq 1_{A}$, or if $2<j<r(\alpha)$; $e_{0}(\alpha, j)=0$ if $r(\alpha)=j=1$ and $\omega(\alpha, 1) \neq 1_{A}$, or if $j=1<r(\alpha)$ and $\omega(\alpha, 1)=1_{A}$, or if $j=r(\alpha)>2$; $e_{0}(\alpha, j)=1$ if $j=1=r(\alpha)$ and $\omega(\alpha, 1)=1_{A}$. Let $E$ be the function from $P$ to $\mathrm{Z}$ given by $E(\alpha, j)=|e(\alpha, j)|+$ $e_{0}(\alpha, j)$ for each $(\alpha, j) \in P$, whence range $(E)=\mathbf{Z}_{>0}$. For nonnegative integers $n$ it will be convenient to let $[n]=\left\{m \mid m \in \mathbf{Z}_{>0}\right.$ and $\left.m<n\right\}$ if $n>0 ;[0]=\varnothing$.

Let $R_{(\alpha, j)}=\{(\alpha, j)\} \times[E(\alpha, j)]$, a subset of $P \times \mathbf{Z}_{>0}$ for which $\left|R_{(\alpha, j)}\right|=$ $E(\alpha, j)$. These $R_{(\alpha, j)}$ 's are mutually disjoint, and the cardinal of the set of nonvoid ones is $\mathfrak{b}$. Thus, $R=\cup_{(\alpha, j)} R_{(\alpha, j)}$, a disjoint union of void and finite sets, has cardinal $\mathfrak{b}$.

Since $|H \backslash J|=\mathfrak{h}>\mathfrak{b}$ there exists at least one injection $\delta$ of $R$ into $H \backslash J$. Write $\delta$ for the induced map, too, and set $X=\delta(R)$ and $X_{(\alpha, j)}=\delta\left(R_{(\alpha, j)}\right)$. These $X_{(\alpha, j)}$ 's constitute a disjoint cover of $X$ by void and finite sets where $\left|X_{(\alpha, j)}\right|=E(\alpha, j)$. If $X_{(\alpha, j)}$ is nonvoid let its members be denoted by the $x(\alpha, j, k)$ 's where the integers $k$ obey $1 \leqslant k \leqslant E(\alpha, j)$. We form a subset $K$ of $J$ by placing all the $u(\alpha, 1)$ in $K$ and all the $v(\alpha, r(\alpha))$ in $K$ for which $e(\alpha, r(\alpha))=0$. Let $Y=(J \backslash K) \cup X$, so that, in particular, $Y \subseteq H \backslash K$ and $K \subseteq H \backslash Y$.

Let us introduce a linear order into each nonvoid $X_{(\alpha, j)}$ by ordering its elements $x(\alpha, j, k)$ with respect to increasing $k$. We can then incorporate such an ordered $X_{(\alpha, j)}$ into a finite cycle $\left(a_{1}, \ldots, a_{n}\right)$ of elements $a_{i} \in H$ by setting $\left(X_{(\alpha, j)}, a_{1}, \ldots, a_{n}\right)=\left(x(\alpha, j, 1), \ldots, x(\alpha, j, E(\alpha, j)), a_{1}, \ldots, a_{n}\right)$ if $X_{(\alpha, j)}$ is nonvoid; just $\left(a_{1}, \ldots, a_{n}\right)$ otherwise.

Let $\operatorname{sgn} e(\alpha, j)=1,0,-1$, according to the sign of $e(\alpha, j)$. Let $\beta_{0}$ be any permutation on $H \backslash Y$. Let $\beta_{1}$ be the permutation on $Y$ that has its d.c.d. defined by the collection $\{C(\alpha, j) \mid(\alpha, j) \in P\}$ of disjoint finite cycles

$$
C(\alpha, j)=(X(\alpha, j), u(\alpha, j+1), v(\alpha, j))^{\operatorname{sgn} e(\alpha, j)}
$$


if either (a) $2<j<r(\alpha)$ or (b) $j=1<r(\alpha)$ and $\omega(\alpha, 1) \neq 1_{A}$;

$$
C(\alpha, j)=(X(\alpha, j), v(\alpha, j))^{\text {sgn } e(\alpha, j)}
$$

if either (c) $j=r(\alpha) \geqslant 2$ and $e(\alpha, r(\alpha)) \neq 0$ or $(\mathrm{d}) j=1=r(\alpha), e(\alpha, r(\alpha)) \neq 0$, and $\omega(\alpha, 1) \neq 1_{A}$;

$$
C(\alpha, j)=(X(\alpha, j), u(\alpha, j+1))^{\operatorname{sgn} e(\alpha, j)}
$$

if (e) $j=1<r(\alpha)$ and $\omega(\alpha, 1)=1_{A}$;

$$
C(\alpha, j)=(X(\alpha, j))^{\operatorname{sgn} e(\alpha, j)}
$$

if (f) $j=1=r(\alpha)$ and $\omega(\alpha, 1)=1_{A}$;

$$
C(\alpha, j)=1
$$

if $(\mathrm{g}) j=r(\alpha)$ and $e(\alpha, r(\alpha))=0$.

One readily computes the length of the cycle $C(\alpha, j)$ to be $|e(\alpha, j)|+1$ whenever $j<r(\alpha)$. But $e(\alpha, j)$ runs over all integers, so that there are finite cycles of arbitrary length in d.c.d. $\left(\beta_{1}\right)$. Thus, $\beta_{1}$ is of infinite order in Sym $Y$. Let $\beta$ be that map from $H$ to $H$ which coincides with $\beta_{0}$ on $H \backslash Y$ and with $\beta_{1}$ on $Y$, so that $\beta \in \operatorname{Sym} H$ and is, like $\beta_{1}$, of infinite order.

Let $A^{\prime}=\langle A, \beta\rangle$ be the subgroup of Sym $H$ on the set $A \cup\{\beta\}$ of generators. Recall that the free group $A^{\#}$ is $\langle A, \gamma\rangle$. Let $\lambda$ be the map from $A^{\#}$ to $A^{\prime}$ given by $\lambda\left(\omega(\alpha, 1) \gamma^{e(\alpha, 1)} \cdots \omega(\alpha, r(\alpha)) \gamma^{e(\alpha, r(\alpha))}\right)=\omega(\alpha, 1) \beta^{e(\alpha, 1)} \cdots \omega(\alpha, r(\alpha)) \beta^{e(\alpha, r(\alpha))}$. Then, $\lambda \in \operatorname{Hom}\left(A^{\#}, A^{\prime}\right), \lambda$ extends the identity map on $A, \lambda(\gamma)=\beta$, and $\lambda$ is onto $A^{\prime}$. If $\omega(\alpha, 1) \neq 1_{A}$ call $u(\alpha, 1)$ the leading vertex for $t_{\alpha}$. If $\omega(\alpha, 1)=1_{A}$ call $x(\alpha, 1,1)$ the leading vertex for $t_{\alpha}$. Then $\lambda\left(t_{\alpha}\right)$, as a permutation on $H$, carries the leading vertex for $t_{\alpha}$ to $v(\alpha, r(\alpha))$, or to $x(\alpha, r(\alpha),|e(\alpha, r(\alpha))|)$, or to $x(\alpha, 1,|e(\alpha, 1)|+1)$, in no case equal to that leading vertex. That is, ker $\lambda$ is trivial, so that $\lambda$ is an isomorphism, and $A^{\prime}=\lambda\left(A^{\#}\right)$ is free on the free set of generators $\lambda(B \cup\{\gamma\})=\lambda(B) \cup\{\lambda(\gamma)\}=\lambda(B) \cup\{\beta\}$, a set of generators of cardinal $\mathfrak{b}$. Since $\lambda$ reduces to the identity map on $A$, this set of generators is just $B \cup\{\beta\}$. Since $\beta \notin A, A^{\prime}$ properly includes $A$. By requiring that $\beta_{0}$ have no fixed points, $\beta$ can be forced to have none either; likewise, $\beta$ can be made to have only finite cycles by so restricting $\beta_{0}$.

If $H$ is countable there are much easier techniques [2] leading to a similar result.

\section{REFERENCES}

1. N. G. de Bruijn, Embedding theorems for infinite groups, Nederl. Akad. Wetensch. Proc. Ser. A 60 (1957), 560-569 = Indag. Math. 49 (1957), 560-569.

2. F. Haimo, M. F. Singer and M. Tretkoff, Remarks on analytic continuation, Bull. London Math. Soc. 12 (1980), 9-12.

3. J. E. Humphreys, Arithmetic groups, Courant Institute of Mathematical Sciences, New York University, 1971.

4. W. Scott, Group theory, Prentice-Hall, Englewood Cliffs, N.J., 1964.

5. M. Tretkoff, A new type of transcendental function, Bull. London Math. Soc. 4 (1972), 167-170.

6. H. Weilandt, Finite permutation groups, Academic Press, New York and London, 1973.

7. __, Unendliche Permutationsgruppen, Zweite Verfielfältigung, York University, 1967.

DePartment of Mathematics, Washington University, St. Lous, Missouri 63130

Department of Mathematics, Stevens Institute, Hoboken, New Jersey 07030 